\title{
Progression of Silicon Solar Cells Luminescence Diagnostic Methods
}

\author{
Radek Stojan*, Jiří Vaněk, Martin Malý \\ Department of Electrical and Electronic Technology, Brno University of Technology, 61600 Brno, Czech Republic \\ *Corresponding Author: xstoja00@stud.feec.vutbr.cz
}

Copyright $@ 2014$ Horizon Research Publishing All rights reserved.

\begin{abstract}
Diagnostic of silicon solar cells defects is permanently one of most important steps in production of solar cells. Specify of diagnostic methods leads to a better understanding and more detailed analysis of manufactured cells. Luminescence methods of solar cells are fast and some of the most common methods today. According to the excitation method of luminescence radiation from silicon solar cells we talk about electroluminescence or photoluminescence methods. Spectral response of using CCD camera with those methods is in band-gap infrared wave length area. The main idea of this paper is to analyze emitted infrared radiation silicon solar cell under the forward bias by polarization spectroscopy. This analysis opens up for potential next new questions in diagnostics defects silicon solar cells by luminescence methods.
\end{abstract}

Keywords Luminescence, Polarization, CCD Camera, Solar Cell

\section{Introduction}

As we mentioned the determination of luminescence in silicon solar cells is an important characterization methods. Solar cells often have different defects which limit their lifetime. Many of these defects can be visualized with luminescence final imaging. The most commonly using technique can be optimized to produce better cells in manufacturing process.

The determination of luminescence in solar cells is an important characterization tool. Typical solar cells often have defects which limit the efficiency or lifetime of the cell. Many of these defects can be visualized with luminescence imaging, so by using this technique the manufacturing process can be optimized to produce better cells.

Electroluminescence (EL) or photoluminescence surveying are a fast characterization methods providing spatially resolved information about electrical, optical and material properties of solar cells.

The intensity of electroluminescence or photoluminescence is proportional to the total excess minority carrier density. All the intrinsic defects and extrinsic deficiencies which reduce the minority carrier density will induce the spatial variation of electroluminescence intensity and be displayed as dark parts in final EL images [1].

The third frequently used method is complementary luminescence emission of micro-plasma. Diagnostics of defect areas can be carried out by measurement of the radiation emitted from the defect during micro-plasma discharge formation.

Using the existing electroluminescence method extended about polarization spectroscopy can be to improve the detection of defects in silicon solar cells.

\section{Materials and Methods}

\subsection{Electroluminescence Characterization}

Electroluminescence is based on the same principle as a light emitting diode. Current is fed into a solar cell (in forward bias) and radiative recombination of carriers causes light emission. It is typical that indirect band-gap semiconductor most of the recombination in silicon occurs via defects in structures. The amount of band-to-band recombination producing radiative emission is relatively low. This radiation can be sensed using an external detector for example CCD camera. Condition for this method is electrical contact and so can only be used once the metallization has been applied and the solar cell is produce complete. Electroluminescence characterization provides a lot of information about the area related uniformity of solar cells and so all modules. Electroluminescence is not destructive characterization method [2].

In our experiments electroluminescence emission due to radiation band-to-band recombination at room temperature of forward-biased crystalline silicon solar cells is surveyed with a CCD grey-scale camera. Neglecting photon recycling, the measured photon current is directly related to the local quasi Fermi-level splitting EFn-EFp according to equation (1) [3]. 
$\Phi_{E L} \infty \int_{0}^{w} U_{r a d} d z=\int_{0}^{w} B p n_{-} d z=n_{i}^{2} \int_{0}^{w} B \exp \left(\frac{E_{F n}-E_{F p}}{k_{B} T}\right) d z$

In equation (1) $\mathrm{W}$ is the thickness of the cell, $\mathrm{B}$ is a material constant, Urad is the total extent of recombination, $\mathrm{dz}$ is the planned length of the cylinder of unit cross section of photon flux, $n i$ is their own carriers concentration, $p$ is concentration of free holes and $\mathrm{n}$ the concentration of free electrons, $\mathrm{k}$ is the Boltzmann constant and $\mathrm{T}$ is the temperature. Radiative recombination coefficient B strongly depends on the free-carrier concentration and decreases considerably with increasing doping concentration.

Electroluminescence has recently been introduced as a tool for investigating the minority- carrier diffusion length for analyzing the rear surface passivation quality of silicon solar cells. For methods electroluminescence and photoluminescence diagnosed cell shows slight radiation (luminescence) with a peak in the infrared spectrum. As shown in Fig. 1, maximum of the emitted radiation is around $1150 \mathrm{~nm}[4]$.

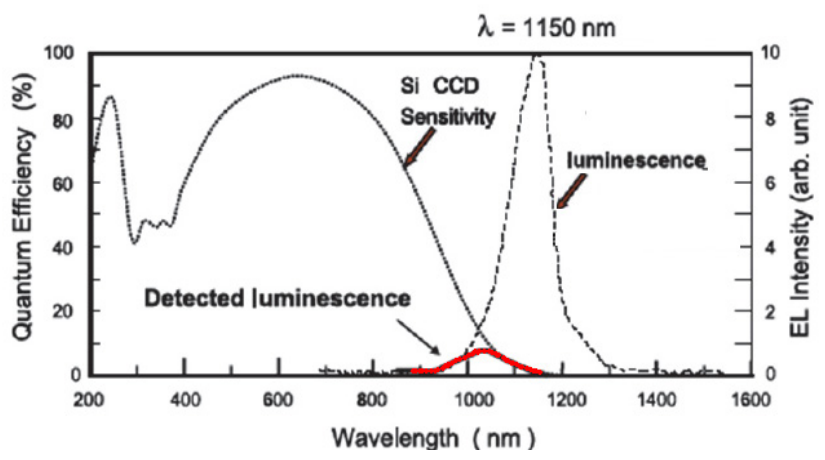

Figure 1. Silicon luminescence with peak $1150 \mathrm{~nm}$ and spectral response of using CCD camera. [1],[4].

\subsection{Photoluminescence Characterization}

Photoluminescence is a kind of luminescence evoked by energy of incident electromagnetic radiation. This one is not destructive characterization method too. Electrical contact is not necessity. According to Stoke's law have an excited radiation of light always higher value of wave length (lower of energy value) than exciting radiation. In material with indirect energy gap structure the third particle is joining the generation recombination process [5].

Photoluminescence spectroscopy is being used to investigate defects and localized band tail states within the band gap of silicon wafers. From the comparison of band-edge photoluminescence intensity and minority carrier lifetime, were confirmed that low photoluminescence intensity regions corresponded to short lifetime regions [5].

\subsection{Micro-Plasma Radiation}

Diagnostics of defect areas can be carried out by measurement of the radiation emitted from the defect during micro-plasma discharge formation. This method is applicable to optoelectronic devices and solar cells. Radiation generated from reverse-biased pn junction defects is used to study local properties. It proves to be useful to measure surface radiation and to make light spots localization to measure the radiation intensity versus voltage plot and its correlation with radiation spectrum [6].

\subsection{Extended Luminescence Methods with Polarization Analysis}

Existing electroluminescence method extended about polarization spectroscopy will produce better analysis of defects in silicon solar cells. Luminescence radiation is characterized as electromagnetic waves. Description of characteristics is described in Table 1 [7].

Table 1. Characteristics of luminescence radiation (electromagnetic wave).

\begin{tabular}{|c|c|}
\hline Characteristics & Descriptions \\
\hline intensity & $\begin{array}{l}\text { number of photons in a given direction passing } \\
\text { through unit area per unit time }\end{array}$ \\
\hline spectrum & $\begin{array}{l}\text { spectral photon flux density per unit interval of } \\
\text { wavelengths or frequencies }\end{array}$ \\
\hline coherence & $\begin{array}{l}\text { given the relationship between the phases of light } \\
\text { waves }\end{array}$ \\
\hline duration & $\begin{array}{l}\text { determined by the inner-lived excited state, from } \\
\text { which there is an issue }\end{array}$ \\
\hline polarization & $\begin{array}{c}\text { direction of oscillation the electric vector } \\
\text { electromagnetic waves }\end{array}$ \\
\hline
\end{tabular}

Designed investigation configuration for extended diagnostic electroluminescence is displayed in Fig.2. It is possible to modify for using electroluminescence and photo luminescence method and then we can also investigate the problematic of polarization luminescence radiation during measurement.

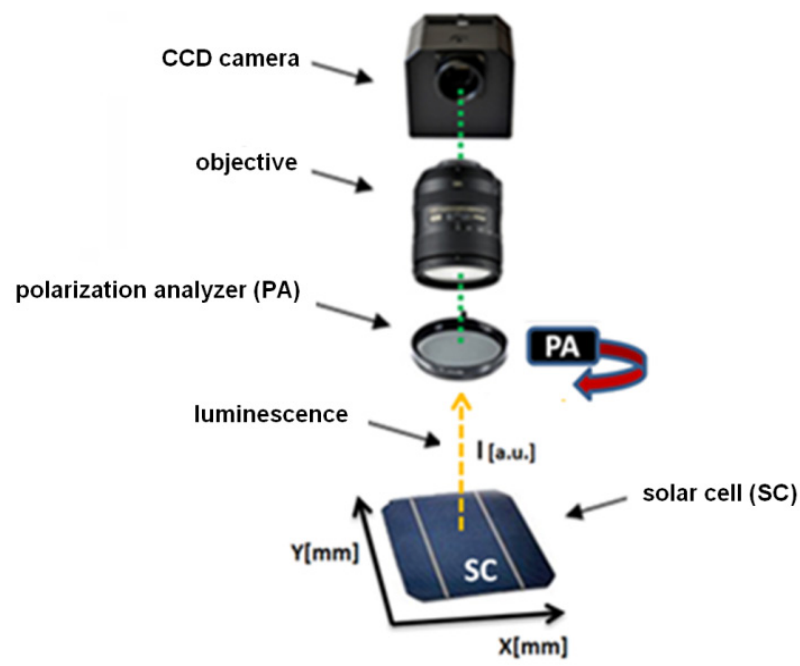

Figure 2. Investigation configuration for extended diagnostic methods electroluminescence.

\subsubsection{Polarization waves}

Polarized light is light in which individual light waves are aligned parallel to one another. It is known that light may be 
polarized partially or completely. The degree of polarization depends on the material (in this case the character of defect) and on the angle at which the light is reflected [8].

Polarized light can pass undiminished through a second polarizer (called analyzer) only if the analyzer's axis of polarization is parallel to the first polarizer. If the analyzer is rotated so that its axis of polarization is at right angles to that of the polarizer, the light is completely blocked [8].

Matthew P. Peloso [9] in their work lists and describes the phenomenon of radiation emitted polycrystalline silicon in electroluminescence displays linear polarization of bar crystal defects - dislocations.

\section{Results}

Investigate poly solar cell with size $125 \times 125 \mathrm{~mm}$ have pseudo square forms with $\mathrm{N}$-type emitter that is diffusion to P-type bulk.

Investigation configuration workplace for testing this method using CCD camera (type G2-3200), which has a spectral response approximately 300 to $1100 \mathrm{~nm}$. The camera has a high sensitivity with a peak quantum efficiency of $80 \%$.

Diagnostics of defect areas can be carried out by measurement of the radiation emitted from the defect during micro-plasma discharge formation (under reverse bias). Micro-plasma radiation we can see in Figure 3. In result image is determine in yellow circle important poly-Si crack defect.

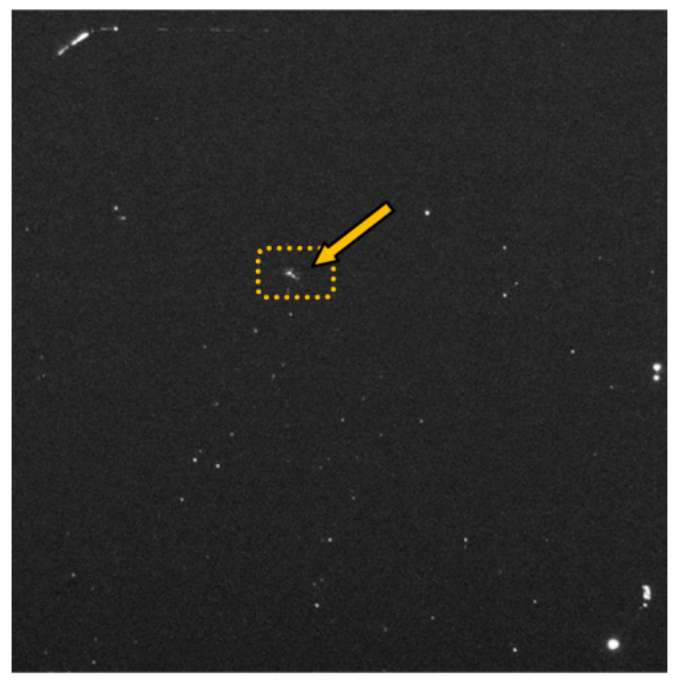

Figure 3. Radiation emitted from the defect during micro-plasma discharge formation.

This wafer was measured by standard electroluminescence testing so (Figure 4). In this result image is the same defect determine in yellow circle too.

Advanced Mathlab analysis of the study defect area can be seen in Figure 5.

In the next time we extend under forward bias the existing electroluminescence technology about polarization spectroscopy and analyzed this solar cell defect. Important cracks defects are displayed in all Figures 3,4 and 5. The actual potential impact of this defect on the electrical solar cell parameters is studied in reference [10].

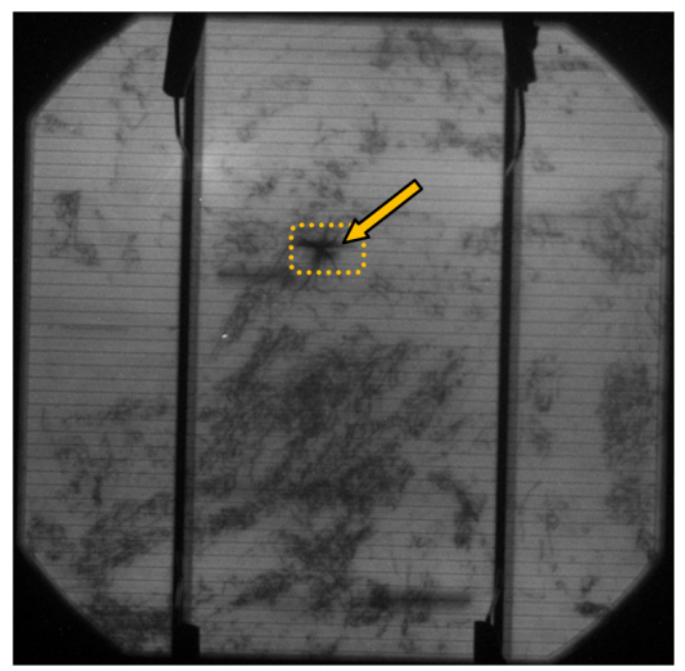

Figure 4. Standard electroluminescence characterization under forward bias and current density about $12 \mathrm{~mA} / \mathrm{cm}^{2}$.

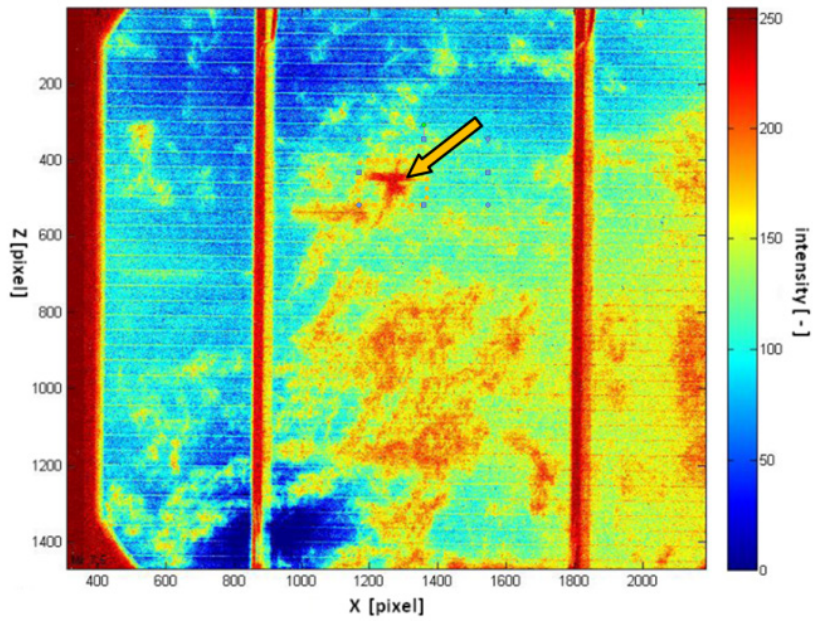

Figure 5. Advanced Mathlab analysis of the study defect area

Factually selected area of poly-Si solar cell sample was measured with a linear polarizing filter. The orientation of the polarizing filter was changed every tenth degree. The angle of rotation was subtracted using precisely defined scale created on the lens CCD camera (type G2-3200). Sample was therefore subjected to 36 measurements (ie. a $360^{\circ}$ circle).

In graph shown in Figure 6, is declared luminescence dependence of the extensive defect in specific determine yellow area. There was sinusoid dependence of the luminescence radiation intensity on the angle of rotation of the polarization analyzer. The intensity of transmitted light by polarization analyzer is dependent on the relative angular position of the polarization plane of luminescence beam and polarizer. In Figure 7 is displayed one of PA position (luminescence peak) by measuring. 


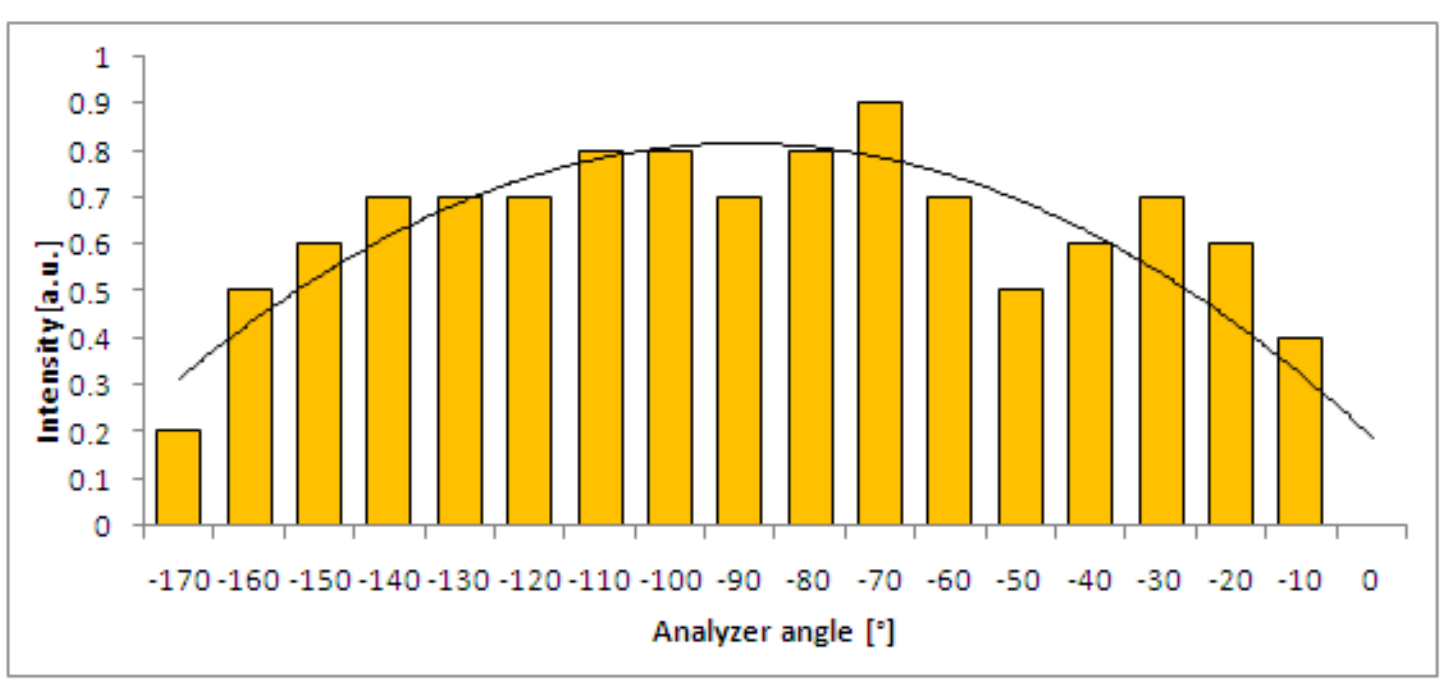

Figure 6. Polarization dependence of luminescence radiation.

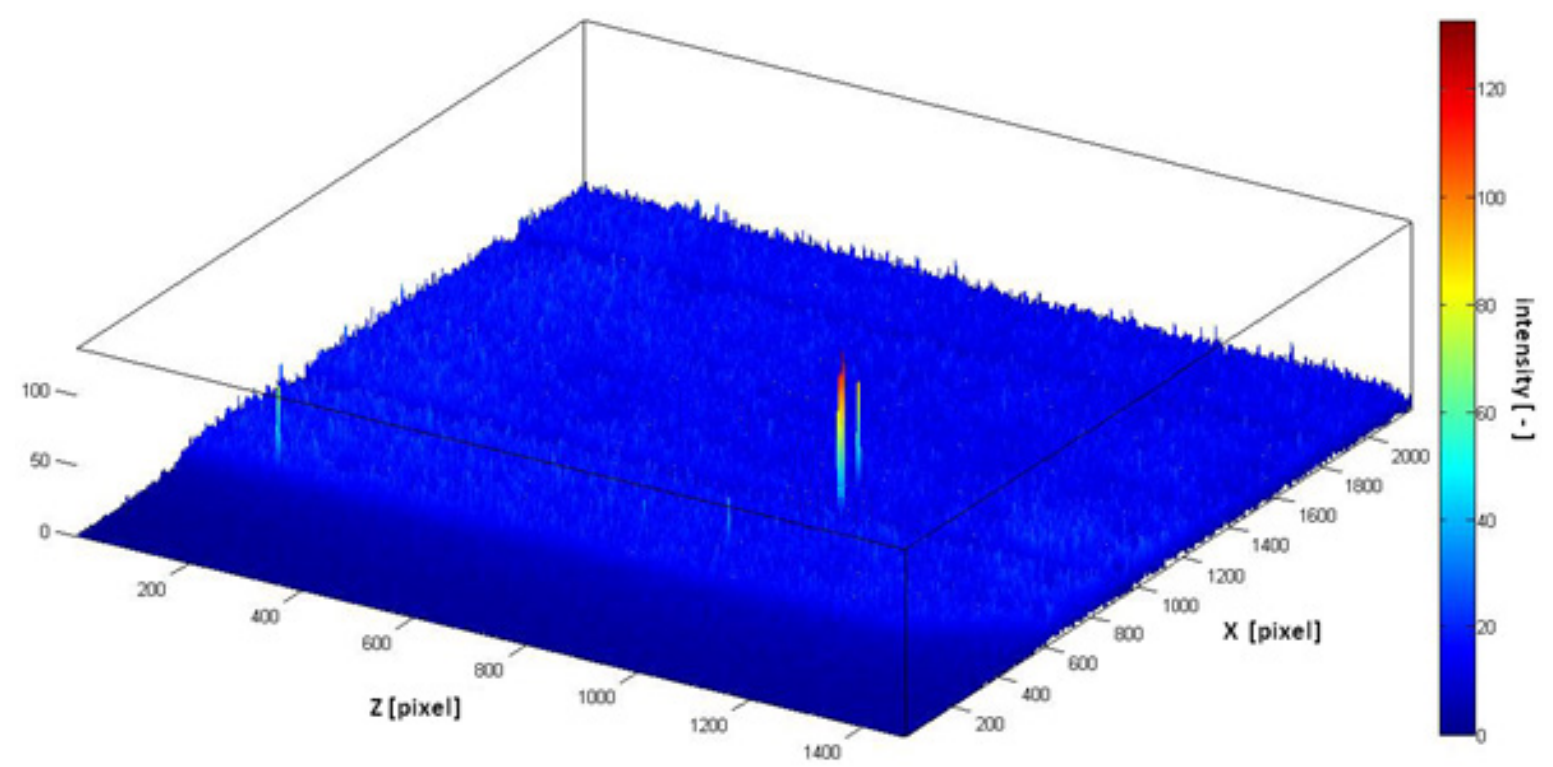

Figure7. Luminescence radiation peak in one of PA position.

\section{Discussion}

In our experiment we showed linear polarization of another type of defect in band-gap wave length. It of course opens up for potential questions. Polarized light can be obtained in various ways, such as by reflection, which leads to a partial polarization and thus that one direction vectors $\mathrm{E}$ prevails. Another way may be refraction, but during refraction is always incomplete polarization.

Defect detection by some of luminescence methods may be hypothetically influence by the interaction of the emitted radiation with the passivation layer. So this is for consideration possible influence of the passivation layer on the nature of waves.

\section{Conclusion}

The precision of detection is therefore a priority for the development of silicon solar cells. Review in this paper can lead to improve the detection of defects in solar cells. We can observed the linear partially polarization luminescence light on poly-silicon crack defect in wave length of spectral response CCD camera. We must add that the used CCD camera is capable of capturing only part of the spectrum of solar cell luminescence about the band-gap energy $(\mathrm{Eg} \approx 1.1$ $\mathrm{eV})$. The researched data subjected to $3 \mathrm{D}$ analysis shows how varied intensity of luminescence radiation depending on angle of rotation of linear polarization analyzer. Sinusoid dependence of luminescence intensity proved this result. The degree of polarization depends on character of defect. It is significant indication of defect type in this measured sample.

Very interesting results from this experiment indicate ways for subsequent experiments. The next not investigated interesting field and technique to characterization silicon solar cell defects is circular luminescence polarization 
characterization.

\section{Acknowledgements}

This research has been subsidized by the project „Materials and technologies for electrotechnics "FEKT-S11-7.

The research is a part of the COST action IC0803, which is supported by the Czech Ministry of Education under grant no. OC09016. The support of project CZ.1.07/2.3.00/20.00 07 WICOMT, financed from the operational program Education for Competitiveness, is gratefully acknowledged. Measurements were performed in laboratories supported by the SIX project; the registration number CZ.1.05/2.1. 00/03. 0072, the operational program Research and Development for Innovation.

Publication of the results was financially supported by the project Popularization of BUT R\&D Results and Support of Systematic Collaboration with Czech Students, no CZ.1.07/2.3.00/35.0004.

\section{REFERENCES}

[1] Fuyuki, H. Kondo, Y. Kaji, A. Ogane, and Y Takahashi, "Analytic Findings in the Electroluminescence Characterization of Crystalline Silicon Solar Cells", J. Appl. Phys. 101, 2007, 023711.

[2] Fuyuki T, Kondo H, Yamazaki T, Takahashi Y, Uraoka Y. Photographic surveying of minority carrier diffusion length in polycrystalline silicon solar cells by electroluminescence. Applied Physics Letters [Internet]. 2005; 86:262108. Available from: http://link.aip.org/link/?APL/ $86 / 262108 / 1$.
[3] J. Dolensky; A. Vesely; J. Vanek and J. Hrozek "Diagnostic methods of solar cells in dependence on temperature", Proc. SPIE7412, Reliability of Photovoltaic Cells, Modules, Components, and Systems II, 74120 U (August 20, 2009); doi: $10.1117 / 12.826135$

[4] Fuyuki, T.; Kondo, H.; Kaji, Y.; Yamazaki, T.; Takahashi, Y.; Uraoka, Y.; "One shot mapping of minority carrier diffusion length in polycrystalline silicon solar cells using electroluminescence", Photovoltaic Specialists Conference, 2005. Conference Record of the Thirty-first IEEE, vol., no.pp. 1343- 1345, 3-7 Jan. 2005 doi: 10.1109/PVSC.2005.1488390.

[5] Pelant,I., Valenta, J.; Luminiscenční spektroskopie, I. Objemové krystalické polovodiče. Praha: Nakladatelství Academia, 2006. 327 stran. ISBN 80-200-1447-0.

[6] Macků, R.; Koktavý, P. Impact of Local Defects on Photon Emission, Electric Current Fluctuation and Reliability of Silicon Solar Cells Studied by Electro- Optical Methods. ElectroScope - http://www.electroscope.zcu. cz, 2011, roč. 2011, č. 2,s. 38-43. ISSN: 1802- 4564.

[7] Malý, Petr. Optika. Vyd. 1. V Praze: Karolinum, 2008, 361 s. ISBN 978-80-246-1342-0.

[8] Kliger, David S.; Lewis, James W.; Randall, Cora Einterez. Polarized Light in Optics and Spectroscopy. United States of America: ACADEMIC PRESS, INC., 1990. 304 s. ISBN 0-12-414975-8.

[9] Matthew P. Peloso; Bram Hoex; and Armin G. Aberle; Polarization analysis of luminescence for the characterization of silicon wafer solar cells. Appl. Phys. Lett. 98, 171914 (2011); doi:10.1063/1.3584857 (3 pages).

[10] Appl. Phys. Lett. 98, 171914 (2011); doi:10.1063/1.3584857 (3 pages). J.I. van Mölken, U.A. Yusufoğlu, A. Safiei, H. Windgassen, R. Khandelwal, T.M. Pletzer, H. Kurz, Impact of Micro-Cracks on the Degradation of Solar Cell Performance Based On Two-Diode Model Parameters, Energy Procedia, Volume 27, 2012, Pages 167-172, ISSN 1876-6102, 10.1016/j.egypro.2012.07.046. 